\title{
A Digital Map Developing Method used in Vehicle Location
}

\section{System}

\author{
Ma Liang
}

College of mechanic and electric engineering, Huanggang normal university,

Huanggang , 438000, Hubei Province, China

44055078@qq.com

Keywords: Vehicle Location, Digital Map, VC++

\begin{abstract}
In this paper we will introduce a method to develop digital maps used in vehicle location. We will introduce how the map was made with $\mathrm{VC}++$. How we describe roads and buildings in the map.

\section{Introduction}

Vehicle location needs digital maps. A digital map contains latitude and longitude information of the roads and buildings. The actual vehicle location system mostly use the digital map developed by professional map company. Use this way, the system has low efficiency, and developers do not have the copyright. Use the digital map developed by yourself can achieve seamless connection between the map and Locating system. And the system will has a high efficiency, and require less map storage space.
\end{abstract}

\section{Getting the map data}

A digital map comprises roads, buildings and open spaces. We should get the latitude and longitude information of those components. There are several ways to get the latitude and longitude information, you can scan a Raster map to turn it to a Vector map; you can use a GPS to get the information and so on. We get the information directly from the National Geographic Information System data base.

\section{Processing the data of the map}

1. Classify the data

The data we get are many points with latitude and longitude coordinates. The elements on the map include: buildings, roads, rivers, green spaces and water. We use several points to represent a road. In a digital map a straight road is represented by a beeline. We can use two points to describe a beeline If there is a flexuous road it will be approximated by many straight roads linked form beginning to end which represented by many beelines. To represent a building or a green space or a water area the traditional way is using a polygon. By getting the latitude and longitude information of the vertexes of the polygon, they can describe a building.

Because the system is used in the vehicle system, the vehicle will only travel on the road. So we should not pay much attention to the outline of the buildings. In this paper, a special method is used to distinguish between different buildings, because most of the buildings are surrounded by roads, we can use the roads around the buildings to distinguish these buildings. We draw the road twice, 
first draw it with a certain pixel width of white line which represent the intervals between the buildings, second draw it with a black line which is narrower than the white line to represents the road itself. If the interval between some buildings has no road, we will see the interval as a special road, when drawing the special road we only draw it once with the white line. So we can describe a building with one coordinate point which represents the central location of the building. The green spaces and water are treated as buildings and rivers as special roads. So it can save a lot of storage space, especially for the portable system, the data storage capacity is not as good as the ordinary PC.

In this paper we use 4 point to describe a road. We define a class named FourPTRoad in VC++. The definition of this class as follows:

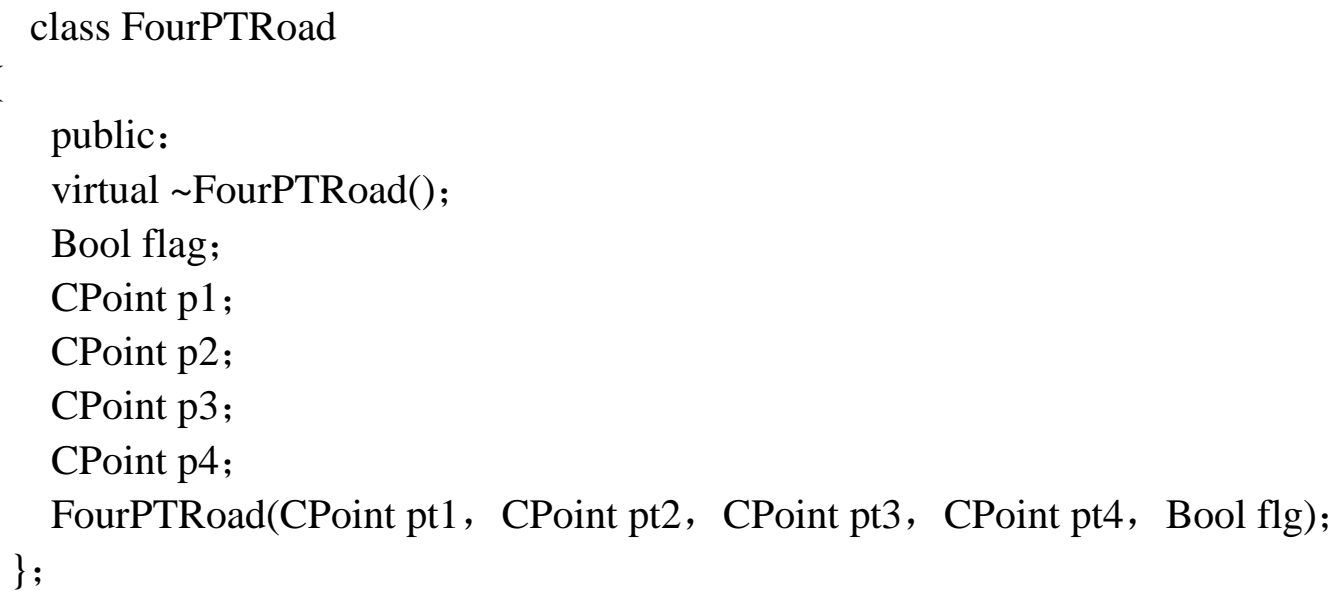

Where: P1, P3, P2, P4 represent 4 points of the road, flag as a symbol, when flag is 0 , means the object is the road, When flag is 1 , means the object is a building interval.

We use one point to describe a building. We define a class named Cbuilding in VC++. The definition of this class as follows:

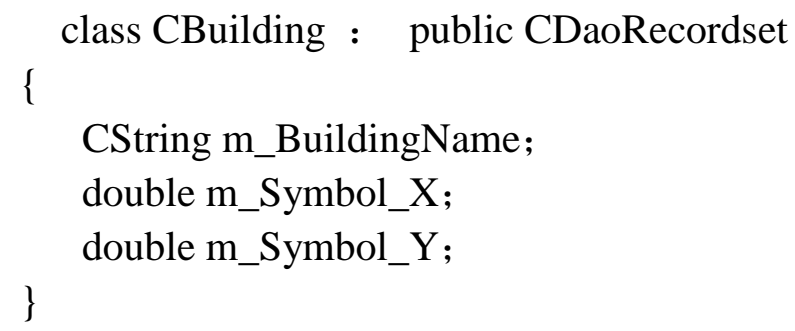

Where: m_Symbol_X, m_Symbol_Y are the latitude and longitude of the point, the $\mathrm{m} \_$BuildingName represents the name of the building.

2. Store the data

Considered the efficiency and the portability We use Access data base to store the data.

\section{Draw the map}

\section{Coordinate transformation}

We want draw an element in a window. The element is described by many points.

The points have coordinates of latitudes and longitudes. But if we want to draw those points in a window we need the pixel coordinates of those points. So we need transform a point's latitude and longitude coordinates to pixel coordinates. 
The latitude and longitude coordinates of a point are $\left(X_{m}, Y_{m}\right)$, and the pixel coordinates of the point are $\left(X_{w}, Y_{w}\right),\left(X_{0}, Y_{0}\right)$ is the latitude and longitude coordinates correspond to the coordinate origin of the window.

The coordinate transformation equation is:

$X_{w}=T \times\left(X_{m}-X_{0}\right)$

$Y_{w}=T \times\left(Y_{m}-Y_{0}\right)$

Where: $\mathrm{T}$ is used to control the map size.

2. Draw the map in window

The program flow chart as Fig 1:

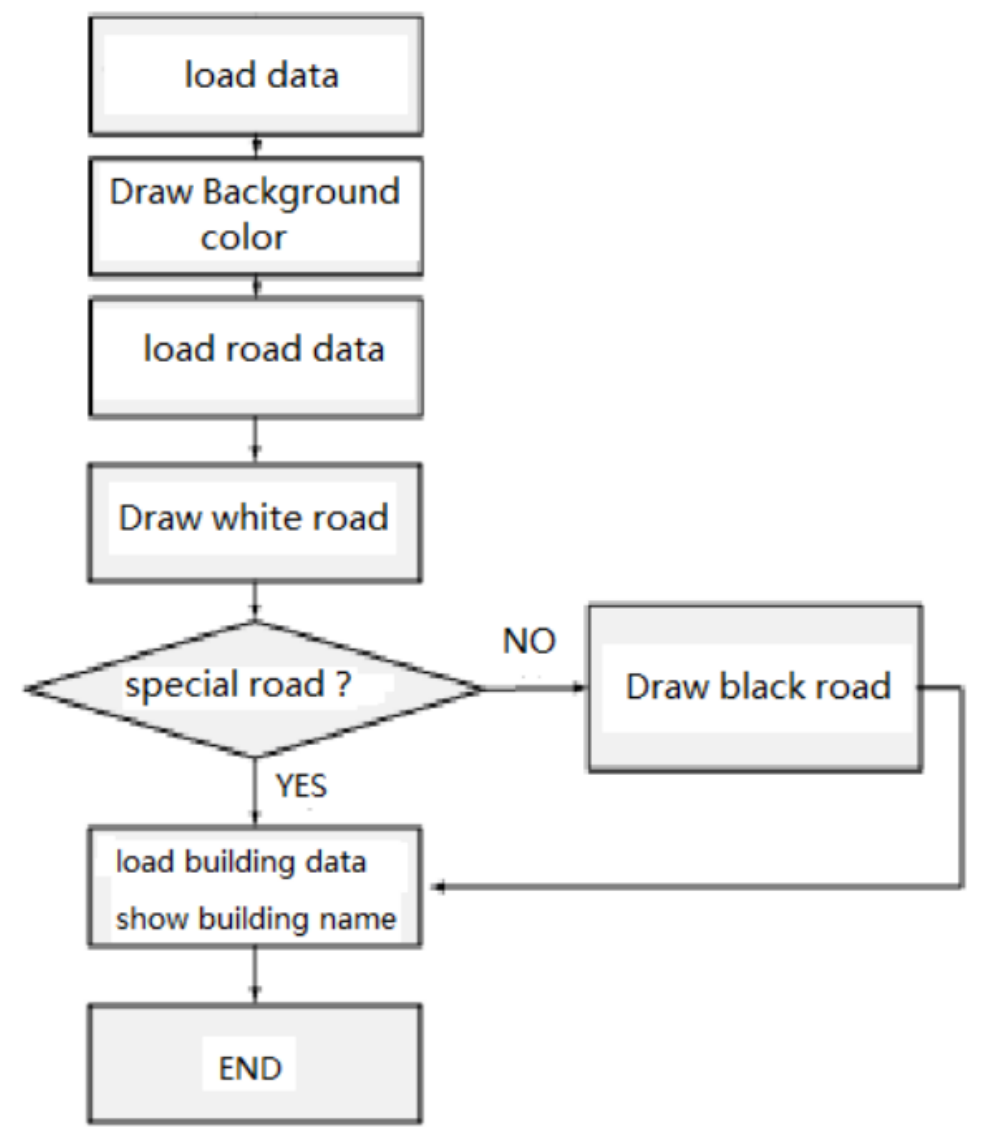

Fig 1: Program flow chart

A map drawn by this method showed in Fig 2: 


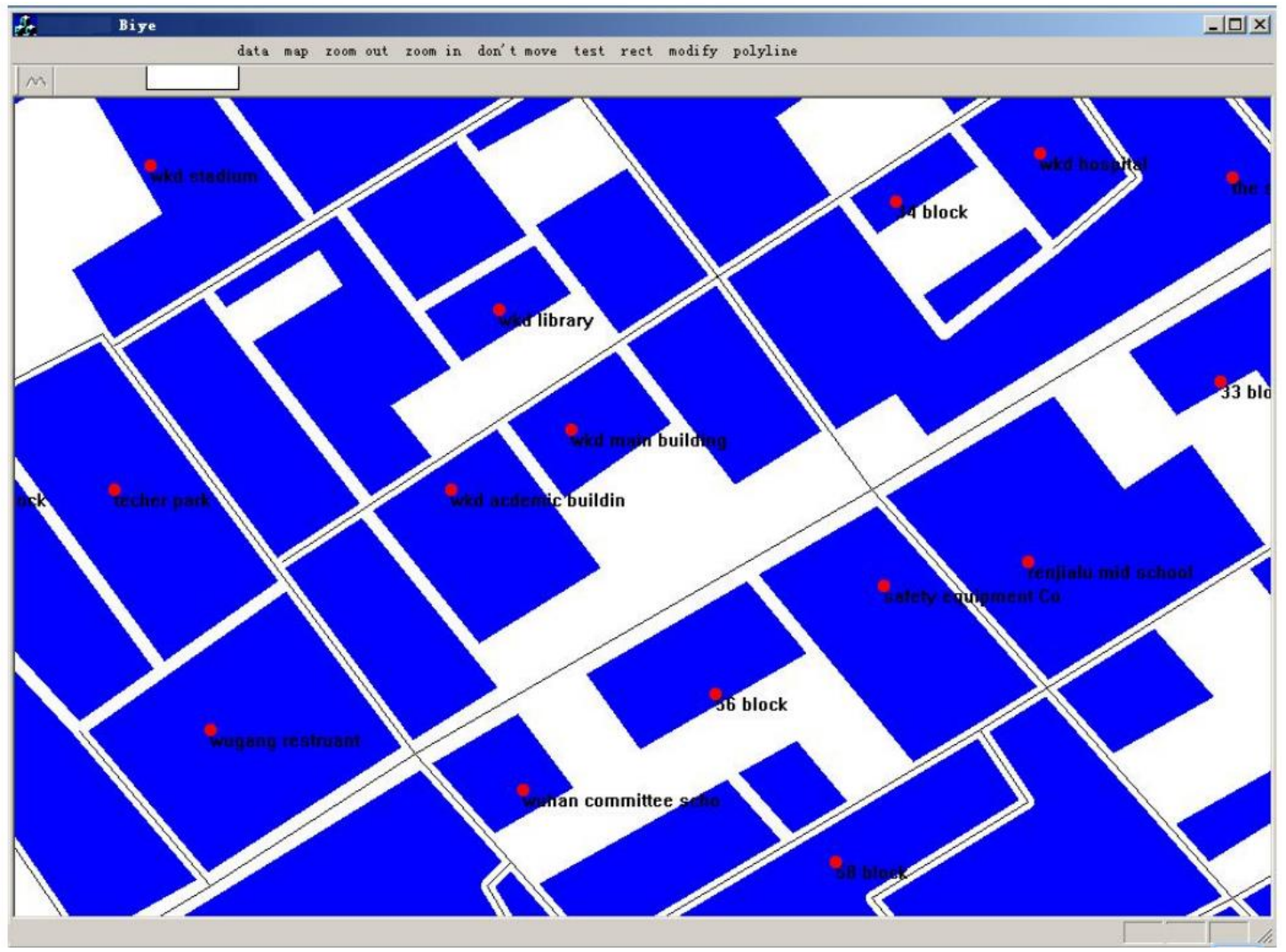

Fig 2: Digital map

\section{Summary}

This paper introduced a method to develop digital maps only for vehicle location. It use special data structure to define the elements of a map, raised the calculating speed, saved a lot of storage space.

Project supported by Huanggang normal university science Foundation (Grant no. 2015022203)

\section{References}

[1]Li Guangfeng, The Research of GPS/GIS in a Car Navigation System, Master Degree Thesis of Northwest University of industry 2003.2

[2]Zhao Yiling, Car Location and Navigation system, China Electronic Industry Publishing House, 1999.4

[3]Min Daiwei, The Research of GPS/GSM in a Car Navigation System, Master Degree Thesis of Northwest University of industry 2003.3

[4] Sinn Kim, Jong-Hwan Kim, Q-factor Map Matching Method Using Adaptive Fuzzy Network[C], IEEE International Fuzzy Systems Conference Proceedings, 1999.

[5] Sinn Kim, Jong-Hwan Kim, Adaptive Fuzzy-network-based C-measure Map-matching Algorithm for Car Navigation System [J]. IEEE Transactions on Industrial Electronics, 2001, (4). 